\title{
Impact-based Piezoelectric Energy Harvester as a Power Source for a Neural Activity Monitoring Circuit
}

\author{
Sunhee $\mathrm{Kim}^{1^{*}}$, Suna $\mathrm{Ju}^{2}$ and Chang-Hyeon $\mathrm{Ji}^{3}$ \\ ${ }^{1}$ Department of System Semiconductor Engineering, Sangmyung University \\ ${ }^{2,3}$ Department of Electronic and Electrical Engineering, Ewha Womans University \\ ${ }^{1}$ happyshkim@smu.ac.kr
}

\begin{abstract}
Recently, energy harvesters have been studied as a power source for implantable biomedical devices. However, practical applications of energy harvesters to implantable medical devices are very rare because energy harvesters and implantable medical devices have been studied separately. In this paper, we present a neural activity monitor circuit, which is one of implantable medical devices, solely powered by an energy harvester. The system consists of an impact-based piezoelectric energy harvester, a power transfer circuit, and a neural signal monitoring circuit. Reliability of the system was verified by comparing it with a system powered by a DC power supply. It was found that the harvester provided sufficient energy to the system for reliable neural activity monitoring. We expect that presented results may work as a reference for designing harvesters and power transfer circuits together with implantable medical devices in an environment of a human body.
\end{abstract}

Keywords: biomedical monitoring, energy harvesting, equivalent circuits, vibration energy

\section{Introduction}

As portable devices, such as wireless sensors, personal information devices and implantable medical devices, have been widely utilized, their power source has become increasingly important. Because they have usually used batteries as their power source and batteries have a limited life, dead batteries must be replaced after a certain period of time. In the case of wireless sensors, using disposable batteries limits service locations and prevents a sensor network from scaling to hundreds or thousands of nodes [1]. In the case of implantable biomedical devices, such as pacemakers [2], electrical stimulators [3], and closed-loop seizure controllers [4,5], battery replacement can cause physical pain, psychological distress, and economic burden for a patient [6, 7]. Recently, energy harvesters have attracted attention because they are expected to replace batteries [8-10].

Energy harvesting techniques have been studied to convert various energy sources, such as solar, thermal energy, acoustic energy, and kinetic energy into electrical energy [11]. Solar energy harvesters have been usually used in wireless sensor network (WSN) because they provide relatively high power density and voltage/current levels required for WSN devices [12]. Thermal energy, bio fuel cells and kinetic energy from body motion have been studied in implantable biomedical fields $[6,13]$. Harvesters based on kinetic energy generated by human-body motion are considered as be more useful in powering implantable devices at this time $[6,13,14]$. Human-motion induced vibration can usually be characterized by relatively low frequency and the random nature [15]. Power Standards Lab shows resonance frequency of human body ranges from $4-5 \mathrm{~Hz}$ to $50-100 \mathrm{~Hz}$

Received (December 19, 2017), Review Result (February 5, 2018), Accepted (February 7, 2018)

* Corresponding Author 
depending on body sections [16]. Ju et al. demonstrated acceleration and frequency measured by using an accelerometer in conditions, walking and running, ranged in the $0.98 \mathrm{~g}-4.05 \mathrm{~g}$ and $1-20 \mathrm{~Hz}$, respectively [15]. Ylli et al. demonstrated impact between shoe and ground during walking and jogging had average acceleration peak ranging in 3$30 \mathrm{~g} \mathrm{[14].}$

Kinetic-energy or vibration-energy harvesters have usually been utilized by using three types of electromechanical transducers: electrostatic [17], electromagnetic [18], and piezoelectric [19]. The piezoelectric energy harvesting technique has been more widely studied than the other two methods because it has many advantages, such as simple configuration, high converting efficiency, high power density, and precise control [11]. Regardless of transducing type, conventional vibration-energy harvesters are effective around their specific frequency [18]. To overcome the issue, various approaches have been developed including multimodal harvesting [20], broadband techniques [21], and nonlinear mechanisms [22]. In this paper, we used a non-resonant piezoelectric energy harvester to harvest energy from low frequency vibrations such as human-body-induced motion [23].

Cadei et al. reported average values of the power consumed by a number of implantable medical devices and the average values of power produced by various harvesters [6]. The reported piezoelectric harvesters generate power between $6 \mathrm{nW}$ and $7.2 \mathrm{~mW}$ (4 piezos). Some implantable medical devices require an average power of between $8 \mathrm{uW}$ and $5.16 \mathrm{~mW}$. These results show that some piezoelectric harvesters can supply sufficient power to implantable medical devices. However, few practical applications have been made of applying energy harvesters to implantable medical devices. In this paper, we propose an actual system that consists of a neural signal monitoring circuit, which is one of implantable medical devices, a power transfer circuit, and an impact-based piezoelectric energy harvester, which powers the circuits, together, as shown in Figure 1.

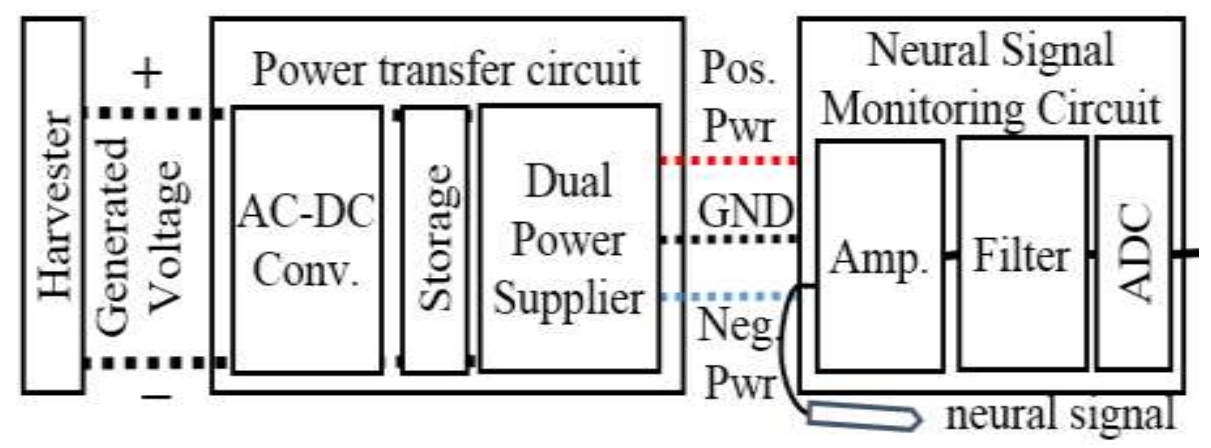

Figure 1. Architecture of a System Powered by a Harvester

\section{Harvester and Circuits}

\subsection{Targeted Harvester}

We previously published a paper on an impact-based frequency-up-converting piezoelectric vibration energy harvester [23]. As shown in Figure 2, the harvester consists of a metal housing, a freely movable ball in the housing and a piezo ceramic based macro fiber composite (MFC) beam. One end of the beam is fixed at the housing and the other, at which two proof masses are attached, is free. The capacitance of the MFC is $12.4 \mathrm{nF}$ and each proof mass is $450 \mathrm{mg}$. The diameter of the spherical ball is $5 \mathrm{~mm}$ and the active area of the MFC is $26 \mathrm{~mm} \times 6.5 \mathrm{~mm}$. The inner volume of the housing is $25 \mathrm{~mm} \times 5.5 \mathrm{~mm} \times 5.5 \mathrm{~mm}$ and the effective volume of the harvester is $26 \mathrm{~mm} \times 10 \mathrm{~mm} \times 8 \mathrm{~mm}$. 


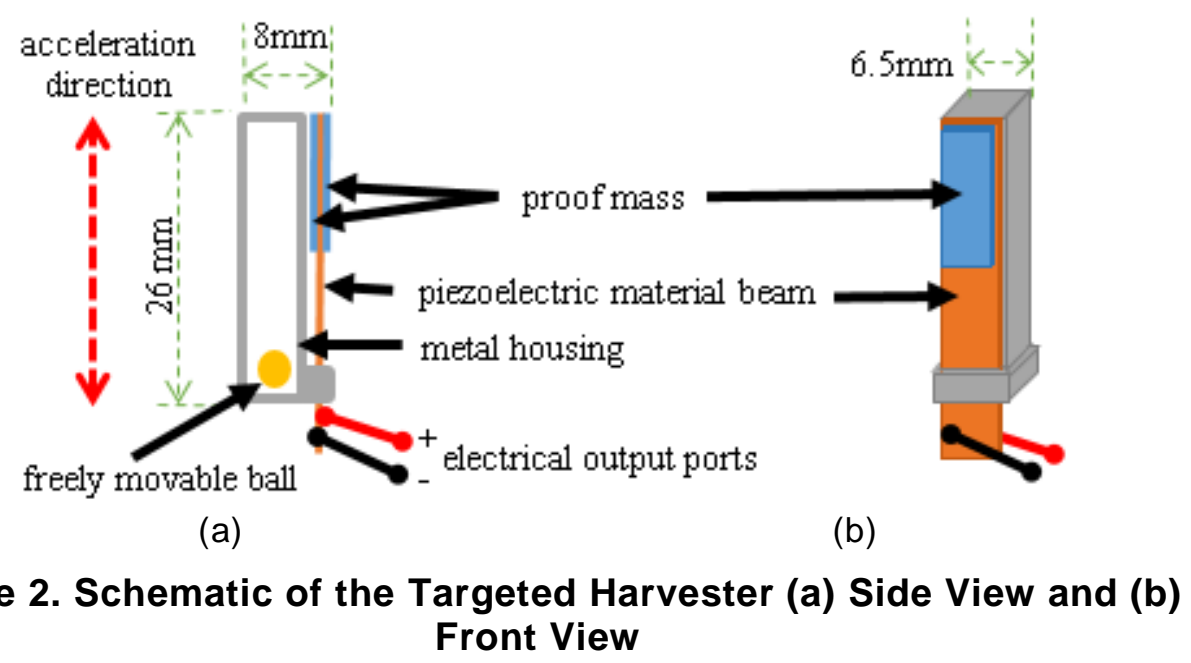

When the harvester shakes, the freely movable ball impacts the housing so that the MFC beam elongates and generates energy. This structure enables separation between frequency of generated energy and frequency of vibration energy. In Ju et al. [23], the harvester was tested at $8 \mathrm{~Hz}$ to $20 \mathrm{~Hz}$ vibrations with accelerations varying from $1 \mathrm{~g}$ to $3 \mathrm{~g}$. Due to the limitation of the used shaker, vibrations with accelerations larger than $3 \mathrm{~g}$ could not be tested. The harvester generated relatively flat frequency response over 2.5 to $3 \mathrm{~g}$ accelerations and maximum open-circuit voltage at $20 \mathrm{~Hz}$ with $3 \mathrm{~g}$ acceleration. As shown in Figure 3, the frequency of the signal generated through impacting was around $8.5 \mathrm{KHz}$. It has the advantage of operating at low frequency vibrations, such as human-body induced vibration, and increasing energy utilization by converting low frequency vibrations into high frequency electrical signals [23, 24].

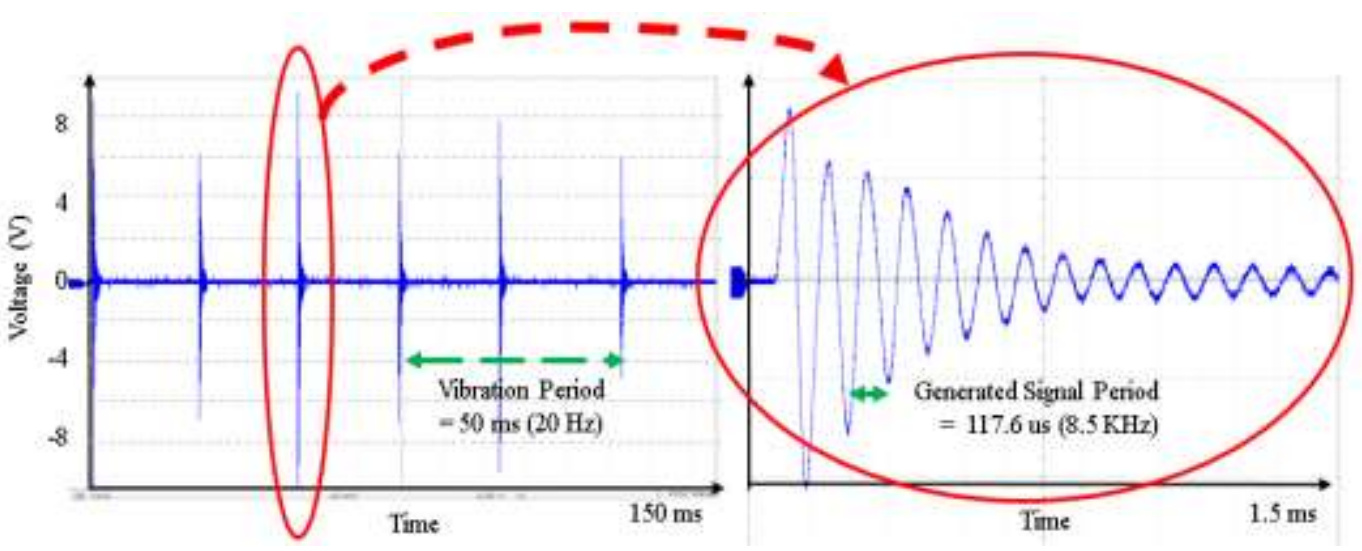

(a)

(b)

Figure 3. Harvester-Generated Signals (a) for $150 \mathrm{~ms}$ and (b) for $1.5 \mathrm{~ms}$

\subsection{Power Transfer Circuit}

Figure 4 shows a power transfer circuit. It can be divided into three two parts: a matching device, a rectifier and a dual power supplier. Because energy generated by harvesters are irregular, a power transfer circuit is needed to supply a suitable DC power. AC-DC rectifiers are usually used for piezoelectric harvesters [25]. Most of them apply an active-switching rectifying structure [26]. These active rectifiers supply higher power extraction efficiency than general passive rectifiers. They, however, require switching control circuits, in other words, external power supply. In addition, most of them assume 
that harvesters generate continuous output signals. In the case of our piezoelectric harvester, the harvester generated sparsely voltages according to vibration frequency as shown in Figure 3. As vibration frequency is lower, a period of no-generated energy is longer and burden of controlling switches is increased. Therefore, we use a general and passive full-bridge rectifier, which consists of four diodes $\left(D_{R}\right)$, to convert irregular voltage to $\mathrm{DC}$ voltage. A capacitor $\left(\mathrm{C}_{\mathrm{S}}\right)$ stores the generated energy. An inductor $\left(\mathrm{L}_{\mathrm{R}}\right)$, as a matching device, connects the harvester to the rectifier to convert energy effectively because the harvester connected to a matching inductor can generate over 2.5 times larger voltage [24]. Although the harvester generated an open-circuit voltage of $16.6 \mathrm{~V}_{\mathrm{p}-\mathrm{p}}$, it did not turn on the full-bridge rectifier without a matching inductor because it did not supply enough current [24].

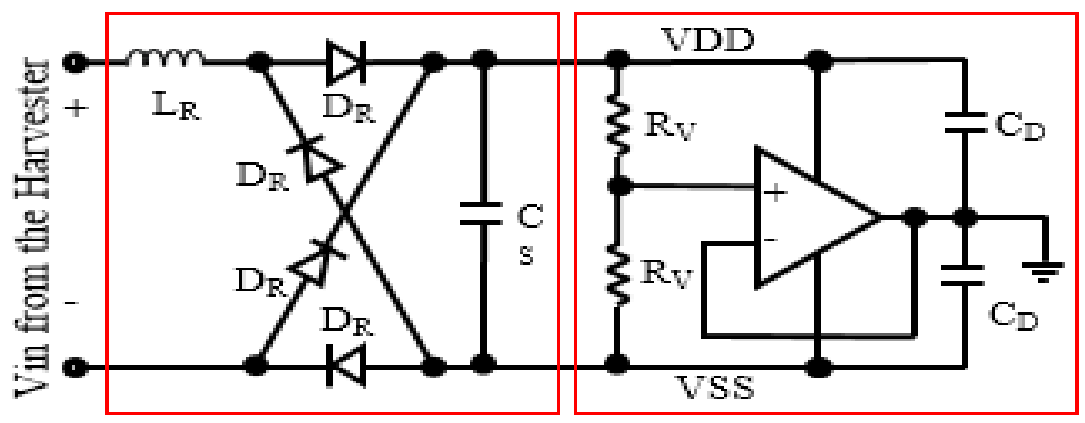

Figure 4. Power Transfer Circuit

The power transfer circuit includes a voltage follower by using an op-amp to supply dual power to the load circuits. A resistor voltage divider (two resistors $\mathrm{R}_{\mathrm{VS}}$ in series) is connected to the generated voltage, of which the high level and low level operate as a positive power supply voltage (VDD) and a negative power supply voltage (VSS), respectively. The divided voltage is directly applied to the non-inverting input terminal of the voltage follower. Because the output voltage is fed back to the inverting input terminal, the output voltage maintains a balanced voltage level between VDD and VSS, even if the load circuits are changed. Two decoupling capacitors $\left(C_{D}\right)$ are placed between the power lines and a ground line.

\subsection{Analog Circuits for Neural Signal Monitoring}

Neural signals have different amplitudes and bandwidths depending on neural types, measuring methods, et al. [27]. The bandwidth and amplitude of various neural signals ranged between $0.05 \mathrm{~Hz}$ and $10 \mathrm{KHz}$ and between $0.02 \mathrm{mV}$ and $10 \mathrm{mV}$, respectively [27]. We focus on intracranial Electroencephalography (iEEG), which has been used to monitor and detect a seizure onset in a seizure controller [28]. Because a human iEEG usually measures local field potentials, the dynamic range of the iEEG is typically limited between $0.1 \mathrm{~Hz}$ and $100 \mathrm{~Hz}$ [27]. The signal amplitude ranges from $0.1 \mathrm{mV}$ to $1 \mathrm{mV}$ [27]. To properly achieve $1 \mathrm{~V}$, for example, the circuits have a total signal gain of more than 1,000 and an ADC with a resolution larger than 6-bit [27].

Most neural signal monitoring circuits consist of amplifiers with enough large gain and filters to receive signals with the required frequency bands. Figure 5 shows the designed analog circuits for the neural signal monitoring of a closed-loop seizure controller. The first stage involves an instrumentation amplifier. It is usually used for an input buffer amplifier because it provides high input impedances, low noise, high common-mode rejection ratio, and low DC offset. We constructed an instrumentation amplifier by using two op-amps and five resistors. The second stage involves a low pass filter (LPF). It is based on an op-amp and a capacitor. It selects a desired low frequency range which is determined by the combination of a resistor $\left(\mathrm{R}_{\mathrm{L} 1}\right)$ and a capacitor $\left(\mathrm{C}_{\mathrm{L} 1}\right)$. The output of the 
LPF is connected to a passive high pass filter (HPF) comprising of components $\mathrm{C}_{\mathrm{H}}$ and $\mathrm{R}_{\mathrm{H}}$. The HPF removes the DC offset. The final stage involves an ADC. It supports a host interface to transfer the sampled data to a host.

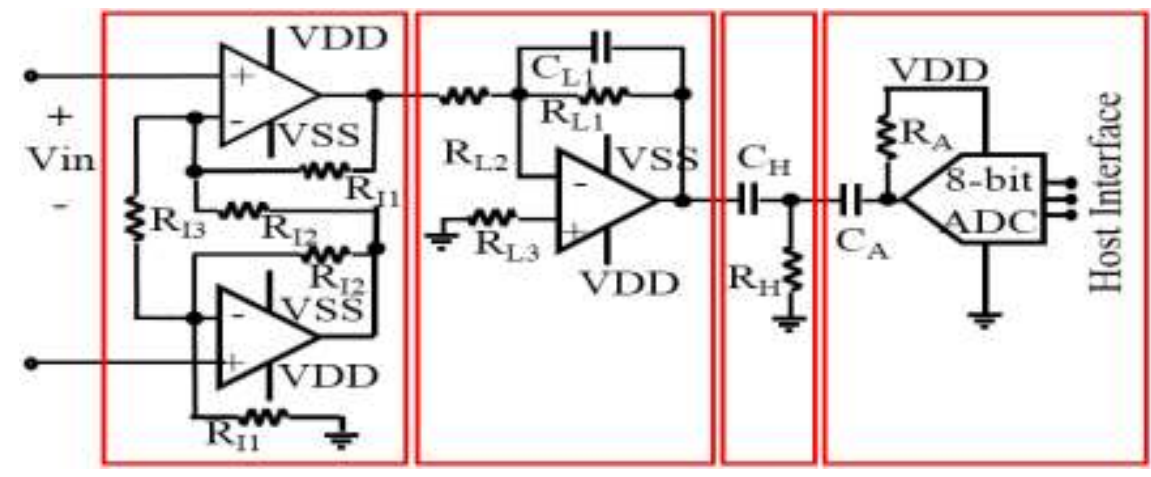

Figure 5. Neural Signal Monitoring Circuit

\section{Test}

\subsection{Prototype}

The rectifier was built using Vishay General Semiconductor SB120 Schottky barrier rectifiers, whose forward voltage and reverse current were $500 \mathrm{mV}$ at $1.0 \mathrm{~A}$ and $0.5 \mathrm{~mA}$, respectively. Analog devices AD8500 CMOS operational amplifiers were used for power transfer circuits and analog circuits. Because the AD8500 can operate from a dual-supply voltage of $\pm 0.9 \mathrm{~V}$ to $\pm 2.75 \mathrm{~V}$, neural signal was connected to input ports without any biasing circuit. A Burr-Brown ADS7868 ADC was used. It is an 8-bit ADC which can operate from a single $1.2 \mathrm{~V}$ to $3.6 \mathrm{~V}$ supply. Because the analog signal input range of the ADC is limited to $0 \mathrm{~V}$ to the supply voltage, biasing circuit components, $\mathrm{C}_{\mathrm{A}}$ and $\mathrm{R}_{\mathrm{A}}$, were added as shown in Figure 5 . The ADC supports an auto power-down mode between conversion cycles and a serial host interface to reduce the power dissipation. Although maximum sample rate of the ADC was $280 \mathrm{kSPS}$, the ADC operated at $1.648 \mathrm{kSPS}$ which was enough to sample neural signal without aliasing. We used a Libertron FPGA Starter Kit including an Altera XC3S200, a universal asynchronous receiver/transmitter (UART) connector, general purpose input/output pins, and a $54 \mathrm{MHz}$ clock oscillator. A serial interface, which controls the ADC, and a UART interface, which transfers the sampled data to a PC, were implemented using the XC3S200. The baud rate of the UART was designed as 115,200.

\subsection{Test Setup}

A shaker system was set up for applying regular vibration force. As shown in Figure 6, the system consisted of an Agilent 33220A waveform generator, an InterM R300PLUS amplifier, and a Brüel \& Kjær LDS V406 M4 shaker. The waveform generator generated a sine wave at a certain frequency. The wave was amplified by the amplifier so that the shaker produced vibrations in a vertical direction at the certain frequency with a chosen acceleration. The harvester was attached and the direction of the acceleration as shown in Figure 2 was aligned with the direction of the vibration. 


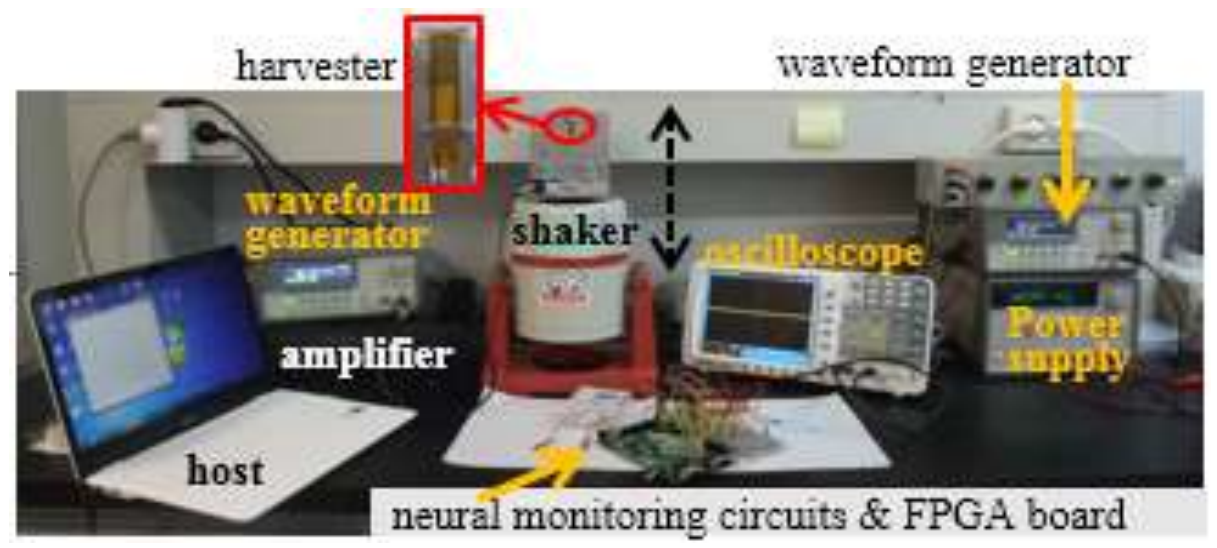

Figure 6. Test System

An input signal of the instrumentation amplifier was generated by an Agilent $33220 \mathrm{~A}$ waveform generator. Voltage waveforms were measured and acquired with an OWON SDS6062 digital oscilloscope and analyzed with an oscilloscope analysis software using a PC. We also used an Agilent E3631 A DC power supply to supply positive and negative supply voltages instead of the harvester. The results were compared with those powered by the harvester.

We set the shaker system to generate vibrations at $20 \mathrm{~Hz}$ with $3 \mathrm{~g}$ acceleration. The input signal of the instrumentation amplifier was a $1 \mathrm{mV}$ sine wave of $50 \mathrm{~Hz}$. The input wave appeared a 2.5 period during one vibration. The storage capacitor $\mathrm{C}_{\mathrm{S}}$ was $1 \mathrm{mF}$ to minimize the output ripple.

\section{Results and Discussion}

Figure 7 shows the supply voltage levels at each stage. As noted above, the targeted harvester generates energy when the ball hits the housing. Therefore, the voltage was generated sparsely and damped as shown in Figure 7(a). The sparse voltage generated by the harvester was rectified and stored at $\mathrm{C}_{\mathrm{s}}$ through the matching inductor and the fullbridge rectifier. As shown in Figure 7(b), the average output voltage of the rectifier was about $5.02 \mathrm{~V}$ without any loads. When the dual power supplier was connected to the rectifier, the VDD and the VSS were $2.68 \mathrm{~V}$ and $-2.80 \mathrm{~V}$, respectively. As the load circuits were added, the magnitudes of the supply voltages decreased. Figure 7(c) shows $2.047 \mathrm{~V}$ VDD and $-2.156 \mathrm{~V}$ VSS when the harvester powered the instrumentation amplifier, the LPF, the HPF, and the ADC as well as the power transfer circuit. The VDD and VSS were changed according to the loads. However, they became stable when the load circuits were determined. They had a generally acceptable output ripple of $+/-1.2 \%$.

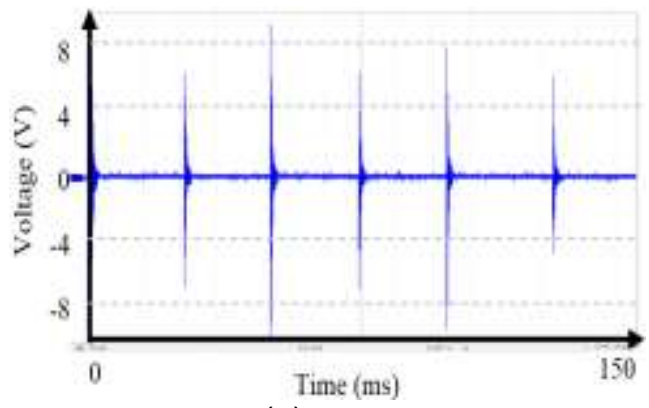

(a)

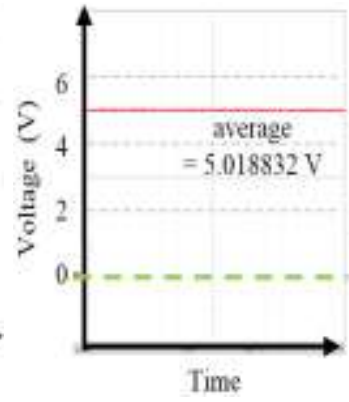

(b)

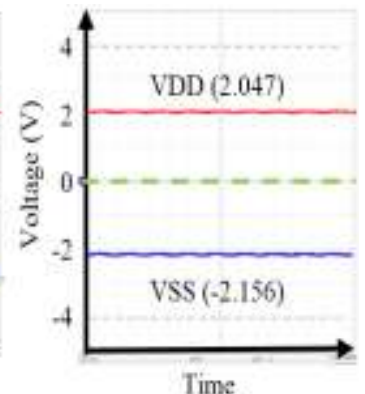

(c)

Figure 7. Supply Voltage Levels (a) Sparse Voltage Generated by Harvester, (b) Voltage across the Storage Capacitor, and (c) VDD and VSS 
Figure 8(a) shows the output voltage signal of the instrumentation amplifier, that is, the input voltage signal of the LPF and the output voltage signal of the LPF. Figure 8(b) shows the output voltage signal of the HPF, the input signal of the ADC and VDD. As shown in Figure 8(b), the input signal of the ADC was shifted to the range of $0 \mathrm{~V}$ to VDD. Because the resolution of the ADC was 8-bit and the input signal of the ADC swung almost in a full range (Figure 8(b)), the sampled ADC output data had a range of about 15 to 240 .

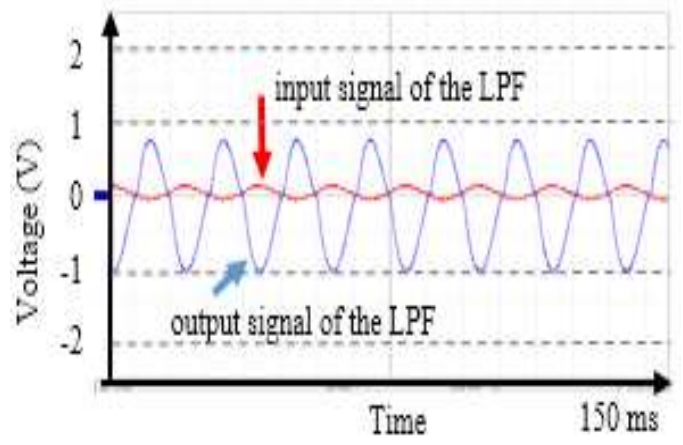

(a)

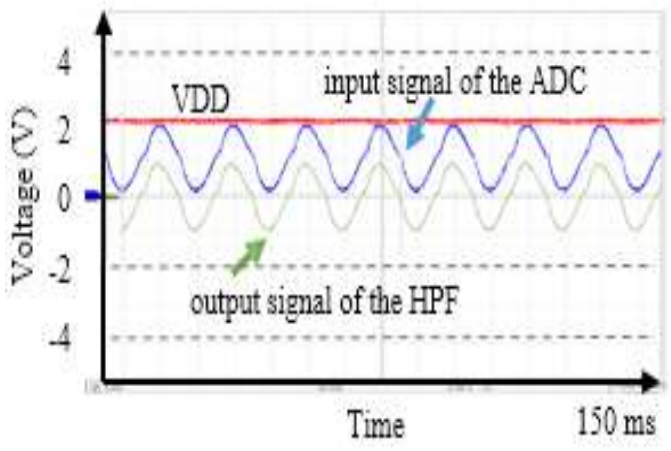

(b)

Figure 8. Data Signals. (a) the Input Signal and the Output Signal of the LPF and (b) the Positive Supply Voltage VDD, the Output Signal of the HPF, and the Input Signal of the ADC

To demonstrate that the harvester can strongly support power to the circuit, we compared the sampled ADC output data, which were transferred to a PC through a UART interface, with the following data. First, we generated a sine wave of $50 \mathrm{~Hz}$. If no noise and distortion were shown, the output data were ideally similar to an amplified sine wave. Second, we performed the experiment again with a power supply. In other words, the power supply was used instead of the harvester, the power transfer circuit, and the storage capacitor as shown in Figure 9. The power supply provided 2 V VDD and -2.1 V VSS, which were the same values as the power levels when the harvester supplied energy to all the circuits.

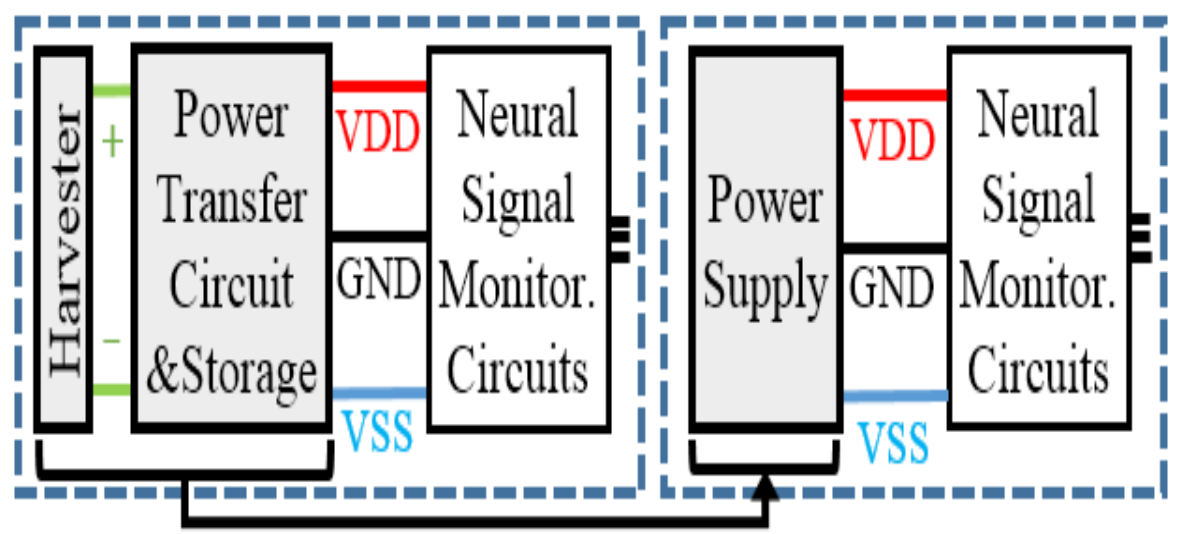

Figure 9. System Architectures: The System was Powered by a Harvester and by a Power Supply

Figure 10 shows the ideal sine wave $d_{i}$ and two UART received data, $d_{h}$ and $d_{p}$, when the circuits were powered by the harvester and by the power supply, respectively. In order to compare data, an error $\varepsilon_{\mathrm{c}}$ was calculated by 


$$
\varepsilon_{c}=\frac{\sum_{n}\left(d_{c, n}-d_{h, n}\right)^{2}}{\sum_{n}\left(d_{c, n}\right)^{2}}, \quad n=1,2, \ldots, N
$$

where $n$ was the number of data and $d_{c}$ was $d_{i}$ or $d_{p}$ according to the comparison data. An error $\varepsilon_{\mathrm{i}}$ was 0.00892 when $\mathrm{d}_{\mathrm{h}}$ was compared with $\mathrm{d}_{\mathrm{i}}$. This error was caused by both noise, such as thermal noise and outside signals, and distortion such as amplitude distortion and phase distortion. These distortions usually occurred due to nonlinearities in the transfer function of active devices and in passive components. Therefore, the error could be reduced by tuning the circuits to minimize distortion. When $d_{h}$ was compared with $d_{p}$, the error $\varepsilon_{\mathrm{p}}$ was 0.00095 , which was much smaller than the error $\varepsilon_{\mathrm{i}}$ calculated previously. In other words, little difference was observed in the circuit performance between when the harvester was used as a power source and when the power supply was used. The result shows that the harvester could generate stable power equivalent to that of a commercial power supply. Therefore, the harvester was considered a good power source in this case.

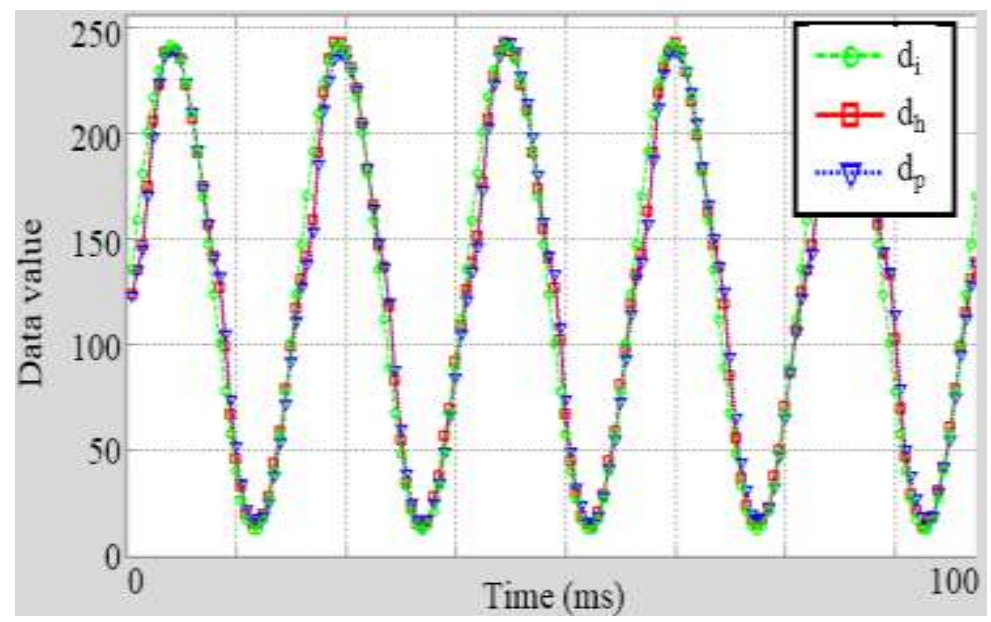

Figure 10. Ideal Sine Wave $d_{i}$ and Two UART Received Data $d_{h}$ and $d_{p}$

To measure the power consumption of the circuits, we changed the storage capacitor to a smaller capacitor. Capacitors store energy and release the stored energy when they deliver energy to the circuits. The energy stored in a capacitor is

$$
\mathrm{E}=\frac{1}{2} C V^{2}
$$

where $\mathrm{C}$ is the capacitance and $\mathrm{V}$ is the voltage across the capacitor. As the capacitance decreases, the stored energy also decreases. Namely, a storage capacitor with very small capacity cannot maintain a stable supply voltage.

Table 1. Average $V_{\max }, V_{\min }, E_{\max }$, and $E_{\min }$ Values According to Loads

\begin{tabular}{|c|c|c|c|c|c|c|}
\hline & \multicolumn{2}{|c|}{$\mathrm{V}_{\max }(\mathrm{V})$} & \multicolumn{2}{c|}{$\mathrm{V}_{\min }(\mathrm{V})$} & \multirow{2}{*}{$\mathrm{E}_{\max }(\mathrm{uW})$} & \multirow{2}{*}{$\mathrm{E}_{\min }(\mathrm{uW})$} \\
\cline { 2 - 5 } & $\mathrm{VDD}$ & $\mathrm{VSS}$ & $\mathrm{VDD}$ & $\mathrm{VSS}$ & & \\
\hline (a): Power transfer circuit & 3.13 & -3.18 & 2.54 & -2.68 & 131.81 & 89.82 \\
\hline (b): (a) + Instrumentation amplifier & 3.11 & -3.10 & 2.38 & -2.53 & 127.52 & 79.56 \\
\hline (c): (b) + LPF and HPF & 3.06 & -3.02 & 2.10 & -2.23 & 121.99 & 61.87 \\
\hline (d): (c)+ ADC & 3.06 & -2.94 & 1.78 & -1.90 & 118.80 & 44.69 \\
\hline
\end{tabular}


Table 2. Power Consumption

\begin{tabular}{|c|c|}
\hline & Power consumption $(\mathrm{uW})$ \\
\hline Power transfer circuit including $\mathrm{C}_{\text {str }}$ leakage & 41.99 \\
\hline Instrumentation amplifier & 5.97 \\
\hline LPF and HPF & 54.14 \\
\hline ADC & 19.97 \\
\hline Instrumentation amplifier + LPF and HPF + ADC & 80.08 \\
\hline Total & 122.08 \\
\hline
\end{tabular}

Figure 11 shows the supply voltage levels when the harvester was shaken at $20 \mathrm{~Hz}$ with $3 \mathrm{~g}$ acceleration, similar to the previous test, but $\mathrm{C}_{\mathrm{S}}$ was $0.33 \mathrm{uF}$. The capacitance of $\mathrm{C}_{\mathrm{S}}$ was small enough to show the voltage change. Figure 11(a) shows VDD and VSS when the harvester powered only the power transfer circuit. Figure 11(b), 11(c), and 11(d) show the VDDs and VSSs when the instrumentation amplifier, the LPF and the HPF, and the ADC, respectively, were added. Each figure shows that the voltage levels increased to the maximum voltage $\mathrm{V}_{\max }$ when a hit occurred, and then decreased to the minimum voltage $\mathrm{V}_{\min }$ until the next hit. Table 1 describes average $\mathrm{V}_{\max }$ values and average $\mathrm{V}_{\min }$ values according to loads at the same case with Figure 11. In addition, it describes average energy before and after the hit, $\mathrm{E}_{\min }$ and $\mathrm{E}_{\max }$, respectively. As more load was added, $\mathrm{E}_{\max }$ was lower because generated energy was same but consumed energy was increased. In addition, the difference between $\mathrm{E}_{\max }$ and $\mathrm{E}_{\min }$ was increased because the energy was leaked and delivered to loads without supply during the decreasing time, i.e. one vibration period $50 \mathrm{~ms}$. We calculated the amount of energy consumed per one vibration period as shown in the following equation,

$$
P=\frac{E_{\max }-E_{\min }}{\tau_{\text {vibr ation }}}
$$

where $\mathrm{T}_{\text {vibration }}$ was one vibration period. Table 2 summarizes the power consumption of each circuit. The power transfer circuit consumed more than half of the total power. This was the consumed energy included storage capacitor leakage as well as delivered energy to the circuit. The neural monitoring circuit, which consists of the instrumentation amplifier, the LPF, the HPF, and the ADC, consumed about a total of about $80.08 \mathrm{uW}$. As a result, the harvester could support $122.08 \mathrm{uW}$ consumption when the harvester was shaken at $20 \mathrm{~Hz}$ with $3 \mathrm{~g}$ acceleration.

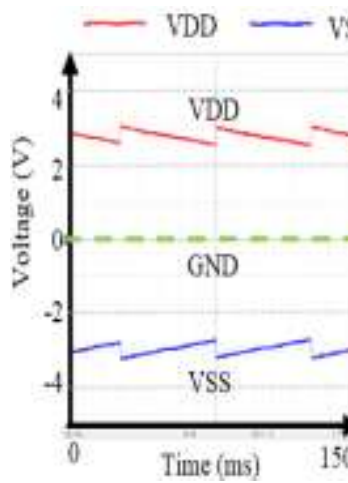

(a)

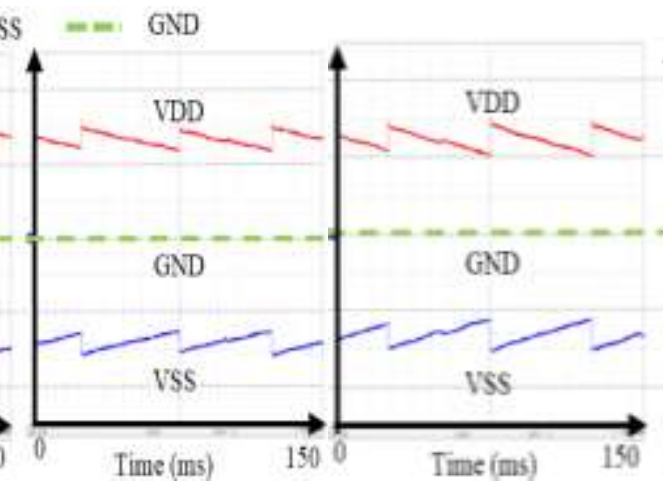

(b)

(c)

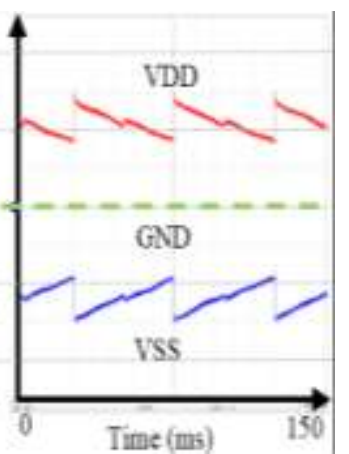

(d)

Figure 11. Supply Voltage Levels, VDD and VSS, According to Loads (a) When the Power Transfer Circuit was Added, (b) When the Instrumentation Amplifier was Added, (c) When the LPF and the HPF were Added, and (d) When the ADC was Added 


\section{Conclusion}

We demonstrate that a piezoelectric energy harvester can supply enough power for the stable operation of the neural signal monitoring circuits of implantable biomedical devices. The piezoelectric harvester can generate energy at low frequency vibrations. An inductor was used to extract power effectively. The full-bridge rectifier was used to convert sparsely generated energy to stable power. The dual power supplier supported a positive power supply and a negative power supply to load circuits. The neural monitoring circuits consisted of the instrumentation amplifier, the LPF and the HPF, and the ADC. The passband of the circuits ranged from $0.1 \mathrm{~Hz}$ to $100 \mathrm{~Hz}$. The ADC converted at $1.648 \mathrm{kSPS}$ with resolutions of 8 bits. The neural monitoring circuits consumed a total of about $80.08 \mathrm{uW}$. The experimental results of the monitoring circuits, which were powered by the piezoelectric harvester, were compared with the experimental results when the circuits were powered by a power supply. This demonstrated that the circuits operate well and the harvester was a good power source in comparison with the power supply. As a result, we found that the harvester could support $122.08 \mathrm{uW}$ consumption when it was shaken at $20 \mathrm{~Hz}$ with $3 \mathrm{~g}$ acceleration.

Although we tested this prototype only at $20 \mathrm{~Hz}$ with $3 \mathrm{~g}$ acceleration, the harvester proved to generate energy in the range of $8 \mathrm{~Hz}$ to $20 \mathrm{~Hz}$ vibrations. Therefore, we expect this prototype can be operated by low frequency and random human-motion vibration. This prototype can be extended to multi-channel monitoring circuits or signal analysis devices. We expect these research results to become a reference for the integrated design of harvesters, power transfer circuits, and implantable medical devices.

\section{Acknowledgments}

This paper is a revised and expanded version of a paper entitled "Piezoelectric Energy Harvester Circuits to Power Neural Signal Monitoring Circuits" presented at Green and Smart Technology (GST 2017), Jeju National University, Jeju Island, Korea and December 1-2, 2017.

\section{References}

[1] Powercast Corporation, "Wireless Sensor Networks", http://www.powercastco.com/applications/ wireless-sensor-networks.

[2] J. Senaratne, M.E. Irwin and M.P. Senaratne, "Pacemaker longevity: are we getting what we are promised?", Pacing and Clinical Electrophysiology, vol. 29, no. 10, (2006), pp. 1044-1054

[3] V. Giagka, A. Demosthenous and N. Donaldson, "Flexible active electrode arrays with ASICs that fit inside the rat's spinal canal", Biomedical Microdevices, vol. 17, no. 6, (2015), pp. 1-13.

[4] M.T. Salam, N. Dang Khoa and M. Sawan, "A low-power implantable device for epileptic seizure detection and neurostimulation”, 2010 IEEE Biomedical Circuits and Systems Conference (BioCAS), Paphos, Cyprus, (2010).

[5] C.P. Young, S.F. Liang, D.W. Chang, Y.C. Liao, F.Z. Shaw and C.H. Hsieh, "A Portable Wireless Online Closed-Loop Seizure Controller in Freely Moving Rats", IEEE Trans. Instrum. Meas., vol. 60, no. 2, (2011), pp. 513-521.

[6] A. Cadei, A. Dionisi, E. Sardini and M. Serpelloni, "Kinetic and thermal energy harvesters for implantable medical devices and biomedical autonomous sensors", Meas. Sci. Technol., vol. 25, no. 1, (2014), 012003.

[7] C. Meng, O.Z. Gall and P.P. Irazoqui, "A flexible super-capacitive solid-state power supply for miniature implantable medical devices", Biomed. Microdevices, vol. 15, no. 6, (2013), pp. 973-983.

[8] S. Almouahed, M. Gouriou, C. Hamitouche, E. Stindel and C. Roux, "The Use of Piezoceramics As Electrical Energy Harvesters Within Instrumented Knee Implant During Walking”, IEEE ASME Trans. Mechatron., vol. 16, no. 5, (2011), pp. 799-807.

[9] L. Fu-To, K. Yu-Chun, H. Jen-Chien, T. His-Yuan, L. Yu-Te and L. Huang-Chen, "A Self-Powering Wireless Environment Monitoring System Using Soil Energy”, IEEE Sensors J., vol. 15, no. 7, (2015), pp. 3751-3758.

[10] M. Magno, N. Jackson, A. Mathewson, L. Benini and E. Popovici, "Combination of hybrid energy harvesters with MEMS piezoelectric and nano-Watt radio wake up to extend lifetime of system for 
wireless sensor nodes", the 26th International Conference on Architecture of Computing Systems (ARCS), Prague, (2013).

[11] A. Erturk, "Electromechanical Modeling of Piezoelectric Energy Harvesters", PhD dissertation, Engineering Mechanics, Virginia Polytechnic Institute and State Univ., Blacksburg, VA, (2009).

[12] K. K. Win, X. Wu, S. Dasgupta, W. J. Wen, R. Kumar and S. K. Panda, "Efficient solar energy harvester for wireless sensor nodes", the 2010 IEEE International Conference on Communication Systems (ICCS), Singapore, (2010).

[13] S. Koul, S. Ahmed and V. Kakkar, "A comparative analysis of different vibration based energy harvesting techniques for implantables", the 2015 International Conference on Computing, Communication \& Automation (ICCCA), Noida, India, (2015).

[14] K. Ylli, D. Hoffmann, A. Willmann, P. Becker, B. Folkmer and Y. Manoli, "Energy harvesting from human motion: exploiting swing and shock excitations", Smart Mater. Struct., vol. 24, no. 2, (2015), 025029.

[15] S. Ju, S.H. Chae, Y. Choi, S. Jun, S.M. Park, S. Lee, H. W. Lee and C. H Ji, "IMPACT BASED PIEZOELECTRIC VIBRATION ENERGY HARVESTER USING SPHERICAL METAL BALL AND MFC", the 7th Asia-Pacific Conference on Transducers and Micro/Nano Technologies, Daegu, Korea, (2014).

[16] Power Standards Lab, "Mechanical Resonances in the Human Body", http://www.powerstandards.com/ HumanResonance.php, (2005).

[17] V. Dorzhiev, A. Karami, P. Basset, F. Marty, V. Dragunov and D. Galayko, "Electret-Free Micromachined Silicon Electrostatic Vibration Energy Harvester With the Bennet's Doubler as Conditioning Circuit”, IEEE Electron Device Lett., vol. 36, no.2, (2015), pp. 183-185.

[18] K. Tao, J. Wu, L. Tang, X. Xia, S. W. Lye, J. Miao and X. Hu, "A novel two-degree-of-freedom MEMS electromagnetic vibration energy harvester", Journal of Micromechanics and Microengineering, vol. 26, no. 3, (2016), p. 035020.

[19] S Roundy and P.K. Wright, "A piezoelectric vibration based generator for wireless electronics", Smart Materials and Structures, vol. 13, no. 5, (2004), pp. 1131-1142.

[20] H. C. Liu, B. W. Soon, N. Wang, C. J. Tay, C. G. Quan and C. Lee, "Feasibility study of a 3D vibrationdriven electromagnetic MEMS energy harvester with multiple vibration modes", J. Micromech. Microeng., vol. 22, no. 12, (2012), p. 125020.

[21] Y. Wang, Q. Zhang, L. Zhao and E. S. Kim, "Non-resonant, broad-band vibration-energy harvester based on self-assembled liquid bearing", the 18th International Conference on Solid-State Sensors, Actuators and Microsystems (TRANSDUCERS). Anchorage, AK, USA, (2015).

[22] K. Tao, S. W. Ley, J. Miao, L. Tang and X. Hu, "Out-of-plane electret-based MEMS energy harvester with the combined nonlinear effect from electrostatic force and a mechanical elastic stopper", J. Micromech. Microeng., vol. 25, no. 10, (2015), p. 104014.

[23] S. Ju, S.H. Chae, Y. Choi, S. Lee, H. W. Lee and C.-H. Ji, "A low frequency vibration energy harvester using magnetoelectric laminate composite", Smart Mater. Struct., vol. 22, no. 11, (2013), p. 115037.

[24] S.H. Kim, S. Ju, C.H. Ji and S.J. Lee, "Equivalent circuit model of an impact-based piezoelectric energy harvester", the 14th International Conference on Micro and Nanotechnology for Power Generation and Energy Conversion Applications, Awaji Island, Hyogo, Japan, (2014).

[25] X.-D. Do, Y.-H. Ko, H.-H. Nguyen, H.-B. Le and S.-G. Lee, "An Efficient Parallel SSHI Rectifier for Piezoelectric Energy Scavenging Systems", the 13th International Conference on the Advanced Communication Technology (ICACT), Seoul, Korea, (2011).

[26] L. Mateu, H. Zessin and P. Spies, "Analytical Method for Selecting a Rectification Technique for a Piezoelectric Generator based on Admittance Measurement", Journal of Physics: Conference Series., vol. 476, no. 1, (2013), p. 012111.

[27] M. S. Chae, Z. Yang and W. Liu, "Microelectronics of Recording, Stimulation, and Wireless Telemetry for Neuroprosthetics: Design and Optimization", Edited Zhou DD and Elias G, Implantable Neural Prostheses 2. Springer-Verlag, New York, (2010), pp. 253-330.

[28] M. Holtkamp, A. Sharan and M.R. Sperling, "Intracranial EEG in predicting surgical outcome in frontal lobe epilepsy”, Epilepsia, vol. 53, no. 10, (2012), pp. 1739-1745.

\section{Authors}

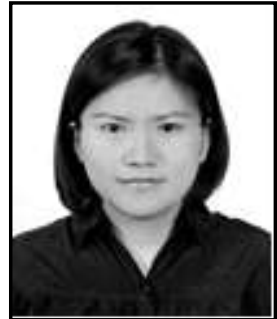

Sunhee Kim, she received the B.S., M.S. and Ph.D degrees in Electronics Engineering from Ewha Womans University, Seoul, Korea, in 2000, 2002 and 2016, respectively. From 2002 to 2005, she worked at Electronics and Telecommunications Research Institute. From 2005 to 2012, she worked at Korea Electronics Technology Institute. She is currently working with the Department of System Semiconductor Engineering at Sangmyung University. Her interests 
include system level low-power design and wireless power transfer technology.

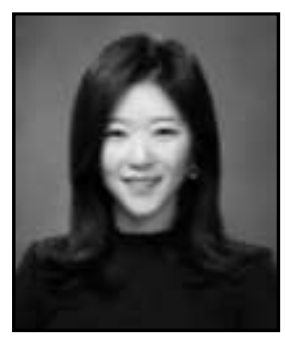

Suna Ju, she received the B.S. degree in electronics engineering and the Ph.D. degree in electronic and electrical engineering from Ewha Womans University, Seoul, Korea, in 2012 and 2017, respectively. Her doctoral thesis concerned the development of piezoelectric vibration energy harvester using indirect impact of springless proof mass. In 2017, she joined the research group for micro and nano systems at the department of electronic and electrical engineering, Ewha Womans University, Seoul, where she is currently working as a Postdoctoral Fellow. Her current research interests include power microelectromechanical systems (MEMS) and energy harvester.

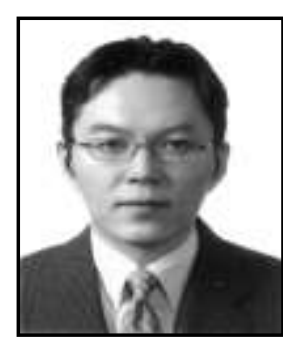

Chang-Hyeon Ji, he received the B.S. and M.S. degrees in electrical engineering and the $\mathrm{Ph} . \mathrm{D}$. degree in electrical engineering and computer science from Seoul National University, Seoul, Korea, in 1995, 1997, and 2001, respectively. His doctoral dissertation concerned the design, fabrication, and testing of electromagnetic micromirrors for microphotonic applications. He was with the LG Electronics Institute of Technology, Seoul, from 2001 to 2006 as a Senior and Chief Research Engineer, where he developed microactuators for various types of applications, including optical communication and raster scanning laser display system. From 2006 to 2011, he was a Postdoctoral Fellow at the Georgia Institute of Technology, Atlanta, where he researched micro power generators and energy harvesters, through-wafer interconnection technology for integrated power electronics, and microfabricated components for wireless power transfer and energy storage. In 2011, he joined the faculty of the Department of Electronics Engineering, Ewha Womans University, Seoul. He is currently working as a Professor at the Department of Electronic and Electrical Engineering. His current research interests include power microelectromechanical systems (MEMS), bio-MEMS, optical MEMS and nanofabrication technology. 